\title{
Linx
}

Revue des linguistes de l'université Paris X Nanterre

$60 \mid 2009$

Nonne scripta manent?

\section{Du Minitel aux SMS, la communication électronique et ses usages pédagogiques}

\section{François Mangenot}

\section{(2) OpenEdition}

\section{Journals}

Édition électronique

URL : http://journals.openedition.org/linx/702

DOI : $10.4000 /$ linx.702

ISSN : 2118-9692

Éditeur

Presses universitaires de Paris Nanterre

\section{Édition imprimée}

Date de publication : 1 mai 2009

Pagination : $97-110$

ISSN : 0246-8743

\section{Référence électronique}

François Mangenot, « Du Minitel aux SMS, la communication électronique et ses usages

pédagogiques », Linx [En ligne], 60 | 2009, mis en ligne le 28 mars 2012, consulté le 29 mai 2019. URL : http://journals.openedition.org/linx/702 ; DOI : 10.4000/linx.702 


\title{
Du Minitel aux SMS \\ la communication électronique et ses usages pédagogiques
}

\author{
François Mangenot \\ Université de Grenoble, Lidilem
}

Ce texte voudrait faire ressortir les apports pionniers de Jacques Anis à l'étude de la communication électronique et des technologies éducatives, tout en évoquant quelques autres voies qu'il avait pressenties mais moins étudiées. Dans un premier temps, on rappellera quelques notions proposées par Jacques Anis, dont celles d'écrit interactif et de communication électronique, et on rappellera le cadre théorique sur lequel il s'appuyait. Ensuite, différentes questions que pose la communication électronique seront envisagées : rapport oral/écrit, effets de l'outil sur la communication, phénomènes identitaires. On abordera enfin quelques enjeux éducatifs liés à l'utilisation des ordinateurs comme moyen de communication, enjeux qu'Anis avait très tôt pressentis.

\section{Une approche sémiolinguistique appliquée à l'écrit interactif}

\section{Quelques notions}

L'expression «communication électronique » est celle que Jacques Anis a retenue, en 2003, pour lancer un projet de recherche à l'intérieur du laboratoire MoDyCo (UMR 7111) et comme titre d'un colloque organisé en février 2004 à l'EHESS avec 
Michel de Fornel et Béatrice Fraenkel ${ }^{1}$. Une des raisons de ce choix terminologique était liée à l'un des objets de recherche qui l'intéressaient depuis le début des années 2000, les SMS (ou textos, of. Anis, 2001a) : la désignation plus classique de « communication médiatisée par ordinateur» (CMO, elle-même traduction de Computer-mediated Communication ou $\mathrm{CMC}^{2}$ ) risquait de ne pas englober un mode de communication fondé non pas sur les ordinateurs mais sur les téléphones portables.

De manière analogue, le Minitel, auquel Jacques Anis s'était beaucoup intéressé vingt ans plus tôt, n'était pas exactement un ordinateur mais simplement un terminal d'un serveur informatique central. A l'époque, Anis et Lebrave (1986) ou Anis (1987) désignaient la communication minitélique par les expressions, qui pouvaient presque à l'époque passer pour des oxymores, «textes interactifs » ou « écrit interactif », expressions justifiées par le fait que l'utilisateur pouvait non seulement recevoir des messages mais aussi en envoyer ; apparaissait alors un «nouveau type de communication écrite quasi immédiate » (Anis et Lebrave, 1986, p. 126). Cependant, l'ambivalence du terme « interactivité » (par ailleurs très galvaudé) était déjà pointée :

Dans la notion - fort ambiguë - d'interactivité, nous distinguerons deux cas. Outre l'interactivité proprement dite, qui concerne la relation entre le système et ses utilisateurs, le vidéotexte comporte en effet une forme moins aisément définissable d'interactivité, qui caractérise les relations établies par le système entre ses différents utilisateurs. (ibid.)

Finalement, « les interactions entre les différents utilisateurs du système » seront considérées comme «l'aspect le plus intéressant [...] dans la mesure où le Minitel a créé $[\ldots]$ une nouvelle forme de communication, qui apparait comme une forme hybride » entre l'écrit et l'oral (op. cit., p. 127). En 2000, Jacques Anis participera à une journée d'étude Notions en questions (à l'ENS Fontenay-Saint-Cloud) au cours de laquelle Bouchard et Mangenot (2001) proposeront de spécialiser les termes interactivité et interaction, le premier pour la « relation» homme-machine, le second pour les interlocutions humaines, reprenant la voie déjà tracée quinze ans plus tôt.

L'objet de recherche sera cerné plus précisément dans (Anis, 1998), ouvrage paru trois ans après l'ouverture d'Internet au grand public en France et considéré par

\footnotetext{
${ }^{1}$ Suite au colloque, un numéro de Langages devait paraitre sous ce même titre, en 2005. Un diaporama, sur le site de Jacques Anis, présente le projet de recherche: http://membres.lycos.fr/jacques92/ (consulté en novembre 2008). Pierozak (2007) évoque le caractère fédérateur du projet, auquel l'auteur de ces lignes était associé.

${ }^{2}$ Champ d'étude interdisciplinaire reconnu dans le monde anglo-saxon, comme en témoigne l'existence depuis 1995 du Joumal of Computer-Mediated Communication (http://onlinelibrary.wiley.com/joumal/10.1111/(ISSN)10836101). La rédactrice en chef de cette revue, Susan Herring, avait d'ailleurs été invitée par J. Anis au colloque des 5-6 février 2004 et s'était montrée étonnée de la vivacité des recherches françaises sur la $\mathrm{CMC}$, qu'elle ignorait totalement. Il faut encore signaler que J. Anis a proposé la traduction française « communication médiée par ordinateur » (plutôt que «médiatisée »), justifiant ce choix par le biais d'une citation de Rachel Panckhurst (Anis, 1999, p. 9) ; c'est cependant « médiatisée » qui l'a finalement emporté dans les usages, comme le prouve une recherche avec Google: 40.100 résultats contre 6070 (à mettre en relation avec 1.140 .000 résultats pour l'expression anglaise, recherche effectuée le $22 / 11 / 2008$, en mettant les expressions entre guillemets).
} 
l'auteur comme son «grand œuvre $»^{3}:$ «Il s'agit d'un écrit né de l'écran et destiné à l'écran. Parmi les diverses applications, nous insistons sur la communication, qui fait surgir un écrit interactif. » (p. 9) et «De nouvelles situations de communication ont fait surgir un écrit interactif et dialogique, qui dispute à l'oral le domaine de l'expression spontanée et de la réaction immédiate. » (p. 269). Si l'on y trouve encore des analyses d'interactions par Minitel (chapitre 3), les nouveaux outils de communication d'Internet y ont également leur place (chapitre 5), comme ils l'auront dans (Anis, 1999a).

\section{Une approche « autonomiste » et sémiologique de l'écrit d'écran}

L'objet précisé, encore fallait-il privilégier telle ou telle démarche d'analyse. Or Jacques Anis avait déjà développé des théories et descriptions de l'écriture, pour paraphraser le titre de son premier ouvrage (Anis, 1988a). Ses objectifs étaient les suivants :

- développer la place de l'écrit dans le champ des sciences du langage, en remettant en question la primauté de l'oral faussement considérée comme un axiome, mais qu'une lecture attentive des textes fondateurs de la linguistique permet de reconsidérer au fond ;

- proposer une approche du système graphique français fondée sur l'autonomie de la forme écrite de la langue ;

- intégrer à la sémiotique textuelle et à la poétique une prise en compte des signifiants et des espaces graphiques, dimension essentielle à la production du sens, qu'on examine des textes littéraires, journalistiques ou utilitaires.

(Anis, 1988a, p. 5)

L'hypothèse autonomiste assigne à la langue une " forme de l'expression graphique » et une «forme de l'expression phonique » corrélées mais distinctes (1988b, p. 213). « La graphématique autonome pointe vers une sémio-linguistique de l'écrit, une graphématique textuelle qui montrera comment l'inscription matérielle du texte contribue à la production du sens » (Anis, 1988b, p. 221). Trois types de signifiants sont distingués, les alphagrammes (lettres avec leurs éventuels diacritiques), les topogrammes (" éléments qui s'ajoutent à la chaine graphique pour rendre plus manifeste sa structuration syntagmatique et énonciative», Anis, 1999b, p. 77) et les logogrammes (signes à valeur idéogrammatique, comme l'arobase, l'esperluette ou les smileys ${ }^{4}$ ).

Appliquée aux écrits minitéliques, puis aux clavardages ${ }^{5}$ sur Internet, cette approche permet à Jacques Anis de dégager une norme spécifique, hybride entre la norme orale et la norme écrite mais néanmoins bien spécifique sur le plan sémiotique :

La norme scripto-conversationnelle est un système d'éléments linguistiques manifestables graphiquement dont la fonction est de réagir à un stimulus donné

\footnotetext{
${ }^{3}$ Voir le site de Jacques Anis.

${ }^{4}$ Le smiley peut cependant être classé tantôt comme un topogramme, tantôt comme un idéogramme (Anis, 1999).

${ }^{5}$ On a choisi ici le terme québécois pour traduire l'anglais « chat», tout en étant conscient que c'est le terme anglais qui prédomine dans les usages en France; le terme officiellement retenu en France - mais très peu utilisé, sans doute à cause de sa connotation surannée - est «causette 》 (JO du 16/3/1999). J. Anis utilisait les termes «chat» ou «tchatche», avec une préférence pour le second (Anis, 1999, p. 9).
} 
François Mangenot

(lequel en règle générale est urgent) d'une manière dynamique, c'est-à-dire d'une manière prompte et immédiate, en exprimant non seulement l'aspect purement communicationnel mais aussi l'aspect émotionnel de l'attitude de l'usager du langage qui réagit. (Anis, 2001c, p. 20)

On reviendra sur cette question très discutée du rapport avec l'oral mais on conclura à ce stade que l'apport original de Jacques Anis est d'avoir le premier posé les bases d'une sémiologie de l'écrit électronique, permettant ainsi à la recherche de dépasser les analyses purement interprétatives et/ou technocentrées.

\section{Quelques questions autour de la CMO}

\section{Hybridation entre oral et écrit}

L'influence respective de l'oral et de l'écrit sur les formes que prend la communication électronique écrite est une question qui revient fréquemment en CMO : « la notion de conversation écrite peut faire problème et rejoint la thématique de l'hybridation entre l'écrit et l'oral développée par plusieurs chercheurs qui se sont intéressés aux langages télématiques »(Anis, 1999b, p. 74). Marcoccia (2000), en s'appuyant sur l'analyse des smileys, avance que la CMO serait calquée sur la communication orale dont elle emprunterait certaines caractéristiques et simulerait celles qu'elle ne peut reproduire : il s'agit là d'une vision peu surprenante chez un chercheur qui tire une bonne part de ses références de l'analyse conversationnelle; quelques années plus tard, une étude plus approfondie de la réception des smileys l'amènera à une position plus nuancée (Marcoccia et Gauducheau, 2007). Pour Anis (1999b, p. 74), «il est sans doute difficile de trancher entre une influence de l'oral sur l'écrit et un rapprochement déterminé par l'élargissement du domaine d'usage de l'écrit». Son approche par la «graphématique des usages» «fait apparaître une plasticité et une hétérogénéité de l'écrit trop souvent méconnues et l'émergence de normes locales » (op. cit., p. 90).

Pour sa part, le linguiste anglais Crystal (2001), dont l'ouvrage Language and the Internet reprend de nombreux points déjà étudiés par Anis $(1998)^{6}$, tente de dégager les spécificités du Netspeak, une langue (ou un langage) de l'Internet qui serait commune à tous les outils de communication écrite en réseau ${ }^{7}$; il adopte une position nuancée :

Globalement, il vaut mieux considérer le Netspeak comme de la langue écrite qui a été tirée d'une certaine façon vers la langue orale que comme de la langue orale qui a été écrite. [...]. Le Netspeak n'est identique ni à l'oral ni à l'écrit, mais fait montre de manière sélective et adaptative des propriétés des deux. (p. 28, notre traduction)

Sa conclusion est que le Netspeak est plus que simplement un hybride de l'oral et de l'écrit; il propose l'équation «Netspeak = écrit + oral + propriétés du média électronique ».

\footnotetext{
${ }^{6}$ Crystal n'avait apparemment pas lu Texte et ordinateur, L'écriture réinventée?

7 «There is a widely held intuition that some sort of Netspeak exists - a type of language displaying features that are unique to the Internet, and encountered in all the above situations, arising out of its character as a medium which is electronic, global, and interactive » (Crystal, 2001, p. 18).
} 
Anis (1998, p. 154-155) souligne par ailleurs à quel point il est difficile d'interpréter certains effets de sens relevant tantôt d'une «oralité simulée » ("Moi j’suis tanguy»), tantôt d'une « subversion du code graphique » (《JEROOOOOOOOOOOOOOOOOOOOOOME !!! »), tantôt du jeu sur la langue (« la 1 devient zobsessionnelle »). Dans (Anis, 1999b, p. 74-75), il souligne que «le partage entre la contamination spontanée et le recours intentionnel à des effets d'oralité est pratiquement impossible à effectuer ».

Un phénomène qui montre en tout cas que l'influence que peuvent avoir l'oral et l'écrit l'un sur l'autre ne joue pas toujours dans le même sens est celui de l'évolution du sigle «lol» («laughing out loud» ou «lots of laugh»), apparu dès les débuts d'Internet (Anis, 2001b, signale sa fréquence dans des corpus recueillis en 1999) et aujourd'hui attesté en France dans le langage oral des adolescents ${ }^{8}$. On observe dans ce cas le passage d'une forme écrite née de la communication électronique vers une forme orale, avec un glissement sémantique : «lol» dans un clavardage signifie en général un rire franc tandis qu'à l'oral il est employé de manière ironique (ce qui est logique puisque le sigle vient alors en concurrence avec le rire). On remarquera que l'équivalent français de «lol », «mdr» («mort de rire »), est moins fréquent chez les francophones et surtout que son emploi à l'oral parait plus difficile pour des raisons phonologiques.

Même la communication écrite asynchrone, telle celle permise par les forums de discussion, peut donner lieu à des variations individuelles importantes à l'intérieur de la même situation de communication. Mangenot et Salam (2010) analysent le registre de langue de trois tuteurs en ligne échangeant avec des apprenants de FLE japonais et montrent des variations par rapport à la norme du français écrit, d'une ampleur variable selon les tuteurs et concernant à la fois le lexique, la morphosyntaxe, la structure des phrases, l'emploi d'interjections et de smileys. Ils concluent :

On observe finalement, à l'instar de Crystal (2001), que l'écriture électronique, même asynchrone, a tendance à tirer l'écrit vers l'oral, sans que cela apparaisse particulièrement choquant, sauf peut-être pour des puristes qui craindraient que les apprenants étrangers ne s'approprient des formes non standard voire erronées. Le langage SMS (Anis, 2001a) porte cette logique beaucoup plus loin en constituant un nouveau code qui, même s'il emprunte la forme écrite, n'a plus grand-chose à voir avec les normes de l'écrit standard. (p. 60)

\section{Effets des outils sur la communication}

La notion d'affordance a été proposée par les chercheurs anglo-saxons en réaction à une vision déterministe de la technologie, afin de mettre en avant le caractère dynamique de la relation entre les propriétés des outils et les actions (ou discours) des utilisateurs :

Les environnements $\mathrm{CMO}$ sont des artefacts qui ne devraient pas être considérés comme des objets non négociables, avec un effet direct et prédictible sur le comportement de l'utilisateur. Leurs caractéristiques sont exploitées et souvent détournées par des

\footnotetext{
8 Observation personnelle confirmée par celles d'autres collègues. Les adolescents en question ne connaissent pas l'origine du sigle. En 2008, LOL a même été utilisé comme titre de film (http://www.lol-lefilm.com).
} 
François Mangenot

utilisateurs faisant des choix actifs et stratégiques. En termes écologiques, les propriétés de la technologie se situent dans une relation dialogique avec les activités des utilisateurs, provoquant des affordances dynamiques. (O’Rourke, 2006, p. 435, notre traduction)

Selon une perspective proche, Crystal (op. cit., p. 24) propose d'observer et analyser les effets des systèmes sur la dimension linguistique :

L'émetteur et le récepteur sont contraints par les propriétés du logiciel et du matériel qui les mettent en relation. Certaines activités langagières traditionnelles peuvent alors être facilitées par ce moyen de communication tandis que d'autres sont rendues impossibles [...]. Comment les utilisateurs réagissent-ils à ces nouvelles pressions et comment compensent-ils sur le plan linguistique?

Jacques Anis, à notre connaissance, n'a jamais fait usage de cette notion d'affordance mais il a toujours été extrêmement attentif aux conditions matérielles de production et de réception textuelles et à leurs effets sur la communication, qu'il s'agisse des limitations du clavier et de l'écran du Minitel, des différences ergonomiques et temporelles entre le canal «Dialogue » ou le canal «Salon» (Anis, 1998), des caractéristiques d'un environnement de type monde virtuel (Anis, 1999b), des « temporalités enchevêtrées aux dynamiques parfois contradictoires » (Anis, 1998, p. 104) du vidéotex ou du clavardage. Il a pu montrer que ces conditions n'avaient pas d'effet automatique : par exemple, le principe d'économie induit par le clavier sommaire du Minitel et par l'urgence temporelle n'empêche pas certains utilisateurs de produire des étirements graphiques (cf. supra) ou des démultiplications de signes de ponctuation, à des fins expressives ; des "profils grapho-linguistiques » (Anis, 1998, p. 157) peuvent alors être dégagés.

Quelques années plus tard, prenant pour objet les «écrits d'écran », Souchier, Jeanneret et Le Marec (2003) proposeront de prendre également en compte la dimension sociale (outre les dimensions technologique et sémiologique), dans une « démarche ethnosémiologique située ». Pour ces auteurs, l'analyse doit combiner trois niveaux différents : «l'objet technique institué comme média d'une part, le formatage du processus de communication par le média d'autre part et, enfin, la façon dont les usagers s'approprient le dispositif» (p. 34). Dans le même esprit, Develotte (2006) a proposé la notion d'«espace d'exposition discursive» pour caractériser la manière dont l'environnement technologique «pré-formate le format et le contenu des échanges » (p. 90).

\section{Phénomènes identitaires}

Anis (1999b, p. 90) avait fait l'hypothèse que chaque environnement suscite l'apparition d'une «variété de langue spécifique participant d'une identité culturelle, se rapprochant parfois du jargon »; il convient dans ce cas d'entendre « environnement » comme référant non seulement au plan technologique mais également au plan social (caractéristiques socioprofessionnelles des interactants, rapports éventuels préexistants, finalité du canal de communication). Dans (Anis, 2004), il analyse la dynamique discursive d'une liste de discussion de spécialistes de la typographie numérique et montre comment un noyau dur d'habitués donne le ton et rejette les écarts. Cela rejoint les observations de Crystal (2001, p. 60-61) : 
Les petites (small-scale) communautés sur Internet vont montrer leur solidarité par des marques d'identité (conscientes ou inconscientes); certaines de ces marques ne seront pas d'ordre linguistique (savoirs partagés, règles morales spécifiques) tandis que d'autres le seront. Les marques linguistiques mettront du temps à évoluer, notamment avec un média où les caractéristiques techniques évoluent aussi rapidement et où un certain degré de non conformisme est monnaie courante, mais un jour ou l'autre elles fourniront à la communauté un dialecte spécialisé que les nouveaux arrivants devront s'approprier s'ils veulent prendre part aux échanges. Un des buts de ce qu'on appellera peut-être un jour la sociolinguistique d'Internet sera d'analyser la systématicité de ces marques et de voir combien de dialectes peuvent être distingués.

Dolci et Spinelli (2007) ont ainsi analysé la manière de fonctionner de petits groupes collaboratifs d'étudiants états-uniens et italiens sur Internet; ils ont pu faire ressortir chez les groupes les plus soudés l'apparition progressive de marques «idioculturelles » tant verbales (au plan du lexique, surtout) que graphiques (smileys spécifiques au groupe).

Dans une approche de type analyse du discours, on privilégie la notion de genre, cherchant à repérer des formes linguistiques (ou plus largement sémiotiques) apparaissant de manière récurrente en lien avec certains fonctionnements sociaux (Charaudeau et Maingueneau, 2002). Plusieurs auteurs (notamment Mourlhon-Dallies, 2007) se sont ainsi interrogés sur les nouveaux genres suscités par la communication électronique. Crystal (2001, p. 14-15), à la recherche d'un Netspeak, se pose la question: «est-ce que le Net est une situation électronique langagière homogène, susceptible de générer une variété unique de langage?». Il répond dans un premier temps qu'il convient d'analyser différentes situations de communication par Internet et relever leurs principales spécificités langagières; il distingue les cinq outils les plus courants (également étudiés dans Anis, 1998, même si ce dernier avait une prédilection pour les modes synchrones), le courriel, les clavardages, les forums, les mondes virtuels, la Toile :

Pour chacune des cinq situations, il est évident que les gens en sont encore à essayer de se raccrocher au potentiel communicatif à leur disposition. Ils sont dans une situation d'apprentissage spéciale. Ils doivent acquérir les règles [...], et cependant il n'y a pas de règles, au sens de modes de comportement universellement acceptés établis par des générations d'usage. (Crystal, 2001, p. 14-15)

Il ne faudrait cependant pas établir un parallèle trop rapide entre l'outil et le genre, parlant par exemple de genre du courriel ou du forum. Mourlhon-Dallies (2007) insiste sur la nécessité de tracer une frontière nette entre genre discursif et support de communication :

Les forums de discussion ne constituent pas à nos yeux un genre : les forums de discussion sur l'environnement sont très différents des forums de passionnés de jeux de figurines ou de supporters de football, au point que nous pensons que le dispositif de communication «forum de discussion » est traversé par différents genres discursifs, adossés à des communautés dont les pratiques d'écriture en ligne et les motifs de recours au forum sont parfois totalement opposés (jeu, convivialité, débat argumenté, échange d'informations). Les «sphères d'utilisation » de la langue en jeu dans ces différents forums paraissent suffisamment dissemblables pour ne pas réunir l'ensemble des réalisations écrites repérées dans ces forums dans un même genre. 
François Mangenot

$\mathrm{Au}$ fond, ne se produit-il pas sur Internet ce qui se produit dans la vie réelle, à savoir la formation de micro-sociétés se dotant de marques identitaires, voire de sociolectes ou, dans le cas d'une certaine stabilisation, de genres de discours? La spécificité serait alors à chercher du côté de la complexité sémiotique du «langage d'Internet », «langage » à plusieurs niveaux : niveau verbal, certes, mais également niveau graphique (néographies, smileys, images), très précisément étudié par Jacques Anis, et enfin niveau ergonomique (fonctionnalités technologiques, temporalité du canal utilisé, permanence de l'écrit) qu'il convient également de prendre en compte pour étudier la manière dont se construisent le sens et la sociabilité. Nous avons pour notre part cherché à prendre ces trois niveaux en compte pour ce qui concerne l'utilisation des forums en tant qu'outils de communication pédagogique. La faculté d'ouvrir des «fils de discussion» dotés d'un titre et la manière dont le système gère ces fils, notamment, nous a paru avoir un effet important sur la structuration des échanges; cette faculté est appropriée de manière différente par différents groupes collaboratifs (Mangenot, 2008a).

A travers certains des exemples fournis, on aura constaté que toutes ces questions de langage, d'appropriation des outils et d'identité se posent également dans le cas des échanges en ligne à finalité pédagogique, échanges qui vont être maintenant abordés de manière plus spécifique.

\section{Enjeux éducatifs de la CMO}

Jacques Anis a consacré une partie de ses écrits aux questions didactiques posées par les technologies de l'information et de la communication, notamment dans leur dimension d'outils de lecture et d'écriture (Anis et Temporal-Marty, 1990 ; Anis, 1995; Anis et Temporal-Marty, 2000). Dès 1986, dans un numéro de Langue française intitulé "Communication et enseignement », il concluait un article intitulé " Ordinateur, pédagogie, communication » par deux réflexions : en tant qu'outil d'enseignement, l'ordinateur affecte les structures de la communication scolaire, de manière spécifique selon le type de logiciel utilisé9. En tant qu'outil de communication, il devrait être utilisé pour "former des hommes capables de vivre en adultes dans un monde "multimédia" » (Anis, 1986, p. 109-110). Douze ans plus tard, après l'apparition d'Internet en France, il conclut son livre par une mise en garde concernant la fracture numérique :

Le rôle de l'école n'a jamais été aussi important: elle doit intégrer au plus vite ces techniques, former les jeunes à ces nouveaux modes du lire-écrire sans écarter les modes traditionnels, développer leur esprit critique face au flux d'informations à classer, sélectionner et évaluer. Il serait catastrophique - et ce risque est loin d'être une pure hypothèse théorique ! - que des outils qui permettent la diffusion la plus rapide et la plus massive du savoir et ouvrent de nouveaux espaces de liberté soient au contraire générateurs d'exclusion et de marginalisation d'une partie de la population. (Anis, 1998, p. 272)

\footnotetext{
${ }^{9}$ Cette piste sera suivie notamment par des chercheurs étudiant les interactions entre pairs devant un ordinateur (Dejean-Thircuir, 2004).
} 
Après avoir mis en avant certaines spécificités de la communication pédagogique médiatisée, on se contentera ici de présenter rapidement deux interrogations qu'elle suscite, celle du tutorat et celle du choix des outils de communication.

Une des principales spécificités de la CMO pédagogique tient à son caractère fortement institutionnalisé et encadré ${ }^{10}$. Les pédagogues assurant cet accompagnement sont le plus souvent appelés «tuteurs», même si ce terme peut recouvrir des statuts disparates, de l'étudiant avancé à l'enseignant chercheur. Une première approche a souvent consisté à mettre à disposition, à côté d'un cours sous forme papier ou multimédia, un forum destiné à poser des questions au sujet du cours; le tutorat est alors dit "réactif», dans la mesure où c'est aux apprenants de prendre l'initiative des questions. Cette forme de tutorat aboutissant souvent à des échanges plutôt rares, on a maintenant parfois recours à une autre approche demandant aux étudiants de réaliser des activités en ligne, avec un tutorat dit "proactif», en ce sens que le tuteur doit veiller à ce que les tâches soient bien réalisées et aider les étudiants à le faire (il arrive même que ce soit le tuteur qui conçoive les tâches, $c f$. Mangenot, 2003).

La situation de communication lors d'un cours à distance fonctionnant selon la seconde approche, notamment quand les participants n'ont jamais l'occasion de se rencontrer, présente alors plusieurs particularités :

- Elle met en présence deux types de participants aux statuts asymétriques : le(s) tuteur(s) d'un côté, les étudiants de l'autre.

- Elle est finalisée par des tâches à accomplir, en général selon un calendrier fixé ; ces tâches font l'objet d'une évaluation et parfois d'une notation. On distingue d'une part les tâches qui amènent simplement les étudiants à mutualiser leurs productions ou à débattre de tel ou tel sujet et d'autre part les tâches collaboratives (Mangenot, 2003; Dejean-Thircuir et Mangenot, 2006): ces dernières impliquent la constitution de groupes restreints (3 à 6) qui, dans un laps de temps déterminé, doivent parvenir à une production commune (pour un exemple, $c f$. Dejean-Thircuir, 2008).

- La communication n'advient le plus souvent que par le canal de la langue écrite; pour qu'un lien social puisse se créer entre les étudiants, ce qui est important pour lutter contre l'isolement (cause d'abandons), il convient d'utiliser un (ou des) outil(s) de communication de type «tous à tous », ce qui exclut par exemple le courrier électronique. Dans le meilleur des cas, on parvient, comme en présentiel, à créer une " communauté d'apprentissage » (Develotte et Mangenot, 2004).

- La communication peut combiner des modes de communication synchrones, comme la messagerie instantanée, ou asynchrones, comme le forum. Le second mode est plus fréquent, car il laisse plus de liberté aux protagonistes. Mais dans le cas de la collaboration en petits groupes, des rendez-vous synchrones sont souvent nécessaires, notamment pour prendre des décisions ( $f$. DejeanThircuir, 2008).

\footnotetext{
${ }^{10}$ Une circulaire du ministère de l'emploi et de la solidarité (DGEFP n ${ }^{\circ} 2001 / 22$ du 20 juillet 2001) précise qu'une formation, quand elle s'effectue à distance, doit faire l'objet d'un accompagnement pédagogique pour être reconnue : «Les modalités d'assistance pédagogique constitueront un élément central d'appréciation de la réalité de la formation. »
} 
François Mangenot

On devine le rôle important que joue le tuteur dans de telles situations. Comme l'a bien exprimé Lamy (2001, p. 142-143),

La gestion de l'interaction en ligne est d'autant plus problématique que les participants n'ont aucun contact présentiel. [...] Il existe un déficit socio-affectif au départ, qu'il est important de compenser. Or le seul «lieu » où puisse intervenir ce rééquilibrage est un lieu purement verbal, et n'est en fait qu'une élaboration textuelle collective. Le pédagogue en ligne doit donc se construire par l'unique biais du discours un rôle nouveau, prenant en compte les propriétés matérielles de l'outil (interface, utilisation de l'espace et du temps) ainsi que les « comportements » autant cognitifs que socio-affectifs d'une communauté d'apprentissage créée elle aussi de toutes pièces par un ensemble de discours.

Il est alors logique qu'un grand nombre de chercheurs aient analysé le rôle et les discours des tuteurs en ligne, afin de faire ressortir la manière dont ils peuvent favoriser les apprentissages. Dans une étude exploratoire portant sur l'ensemble des échanges (1074 messages) liés à un cours à distance proposé via Internet, Celik et Mangenot (2004) étudient par exemple la structure des interactions, pour la comparer avec la structure ternaire souvent mise en évidence dans les interactions en classe présentielle; ils analysent ensuite les marques énonciatives permettant de repérer l'établissement d'une communauté d'apprentissage ; ils cherchent enfin à caractériser ces interactions pédagogiques en ligne en les comparant à d'autres genres de discours, comme ceux générés par les travaux dirigés présentiels, la formation à distance traditionnelle ou la communication électronique non pédagogique. De manière plus pointue, Celik (2008) analyse le discours de deux tuteurs (par ailleurs enseignants chercheurs) d'une mâtrise de FLE en ligne, pour montrer à quel point chacun des deux, d'une manière différente, joue à la fois sur le plan socio-affectif et sur le plan socio-cognitif pour «faire naitre une dynamique de groupe qui doit conduire les étudiants à une réflexion partagée. [...] Grâce à l'écrit, ils ont construit un personnage qui est devenu le médiateur entre leur statut de professeur et les étudiants, un peu à la manière de ces avatars que certains didacticiels font apparaître sur l'écran, mais avec bien davantage de finesse. »

En ce qui concerne le choix des outils de communication, une approche sémio-pragmatique permet de faire ressortir les possibilités et les limites (les affordances, cf. supra) des différents outils disponibles, ce qui peut aider à leur sélection et guider leur utilisation en vue de tel ou tel objectif pédagogique : clavardage (Kern, 1995), forums (Mangenot, 2004 ; 2008), blogs (Soubrié, 2006 ; 2008), plateformes audiosynchrones (Lamy, 2006), etc. Le blog, par exemple, est un outil hybride entre le site et le forum : comme le site, mais plus facilement, il permet à un individu de devenir auteur de contenus; la possibilité d'ajouter régulièrement des billets (pour l'auteur) et des commentaires à ces billets (pour les visiteurs) l'apparente par contre aux outils plus conversationnels comme le forum (Soubrié, 2008). Une utilisation pédagogique des blogs doit tenir compte de ces caractéristiques. La notion de «scénario de communication », définie comme une planification des échanges en ligne que l'on cherche à susciter (Mangenot, 2008b), permet d'attirer l'attention sur le caractère systémique des choix qu'un concepteur doit effectuer en ce qui concerne cette planification : choix des outils, avec leurs spécificités sémiologiques, des paramètres de travail collectif, des objectifs sociaux et communicationnels, de la temporalité des échanges. 
Du Minitel aux SMS : la communication électronique et ses usages pédagogiques

\section{Conclusion}

Dans de nombreux domaines liés aux TIC et à la communication électronique, Jacques Anis était un visionnaire et a joué un rôle de pionnier (Gadet, 2007). Grâce notamment à l'exception française du Minitel, il a pu, bien avant qu'Internet ne devienne un réseau grand public, analyser les caractéristiques d'un nouveau mode de communication hybride qu'une grande partie de la population utilise aujourd'hui. Il a également très vite perçu les potentialités de l'outil informatique mis au service de l'apprentissage de la lecture écriture et de la communication pédagogique. A la fin de sa vie, il était sur le point de réussir à fédérer, dans son projet « La communication électronique », la plupart des chercheurs français s'intéressant à la CMO, pédagogique ou non ( $f$. Pierozak, 2007) qui partagent, comme cet article l'a esquissé, nombre de préoccupations communes. 
François Mangenot

\section{RÉFÉRENCES BIBLIOGRAPHIQUES}

ANIS, J., LEBRAVE, J.-L., 1986, «Des textes interactifs? », LINX n 14, Langue et machines, CRL de Paris X-Nanterre, p. 107-131.

ANIS, J., 1986, «Ordinateur, pédagogie, communication », Langue française $\mathrm{n}^{\circ}$ 70, Communication et enseignement, p. 98-111.

ANIS, J., 1987, «Le vidéotex interactif : un nouvel espace-temps pour la communication écrite », LINX $\mathrm{n}^{\circ} 17$, Le texte et l'ordinateur, p. 46-55.

ANIS, J., 1988a, avec la collaboration de Jean-Louis Chiss et Christian Puech, L'écriture : théories et descriptions, Bruxelles, De Boeck-Wesmael.

ANIS, J., 1988b, «Une graphématique autonome ? », in Catach, N. (éd.) Pour une théorie de la langue écrite, Paris, Editions du CNRS, p. 213-223.

Anis, J., TemPoral-Marty, N., (eds.), 1990, Écriture, informatique, pédagogies, Paris, CNDP.

ANIS, J., 1995, «Traitement de texte et méta-écriture », Repères n 11, Paris, INRP, p. 15-27.

ANIS, J., (ed.), 1999a, Internet, communication et langue française, Paris, Hermès.

ANIS, J., 1999b, « Chats et usages graphiques », in Anis, J., p. 71-90.

ANIS, J., MARTY, N., (eds.), 2000, Nouvelles technologies et lecture-écriture, Paris, CNDP.

ANIS, J., 2001a, Parlez-vous texto?, Paris, Le Cherche-Midi.

ANIS, J., 2001b, « Approche sémiolinguistique d’interactions écrites en temps réel sur l'internet », in Bouchard, R. et Mangenot, F. (eds.), p. 147-168.

ANIS, J., 2001c, «Approche sémiolinguistique des représentations de l'ego dans la Communication Médiée par Ordinateur », Langages n ${ }^{144}$, Paris, Larousse, p. 20-38.

ANIS, J., 2004, « La dynamique discursive d'une liste de diffusion : analyse d'une interaction sur typographie@irisa.fr », Les Carnets du Cediscor nº 8, p. 39-56.

Bouchard, R., Mangenot, F., (eds.), 2001, Interactivité, interactions et multimédia, Collection « Notions en question ", Lyon, ENS-Editions.

CeliK, C., 2008, "Analyse de pratiques de tutorat dans un campus numérique de maitrise de français langue étrangère à distance », Apprentissage des langues et systèmes d'information et de communication Vol. 11. Revue en ligne : http://alsic.org

Celik, C., MAngenot, F., 2004, «Caractéristiques discursives de la communication pédagogique par forum », Les Carnets du Cediscor n 8, p. 75-88.

Charaudeau, P., Maingueneau, D., (eds.), 2002, Dictionnaire d'analyse du discours, Paris, Seuil.

CRYSTAL, D., 2001, Language and the Internet, Cambridge University Press.

DejeAn-Thircuir, C., 2004, Modalités de collaboration entre pairs devant un ordinateur. Etude pragmatique et didactique d'une activité de rédaction collective en français langue étrangère, Thèse de doctorat, Université Stendhal-Grenoble 3.

Dejean-Thircuir, C., Mangenot, F., 2006, «Tâches et scénario de communication dans les classes virtuelles », Les Cabiers de l'Asdifle n 17, p. 310-321. 
Dejean-Thircuir, C., 2008, «Modalités de collaboration entre étudiants et constitution d'une communauté dans une activité à distance ", Apprentissage des langues et systèmes d'information et de communication Vol. 11. Revue en ligne : http://alsic.org

Develotte, C., 2006, «Décrire l'espace d'exposition discursive dans un campus numérique », Le français dans le monde, Recherches et applications $\mathrm{n}^{\circ} 40$, Les échanges en ligne dans l'apprentissage et la formation, p. 88-100.

Develotte, C., Mangenot, F., 2004, «Tutorat et communauté dans un Campus numérique non collaboratif », Distances et savoirs Vol. 2 n 2-3, p. 309-333.

DOLCI, R., SPINELLI, B., 2007, « La dimension idioculturelle des micro-communautés d'apprentissage en ligne ", Lidil n ${ }^{\circ} 36$, Echanges exolingues via Internet et appropriation des langues-cultures, p. 69-92.

GAdET, F., 2007, «À la mémoire de Jacques Anis », Glottopol 10, Regards sur l'internet, dans ses dimensions langagières, Penser les continuités et discontinuités, revue en ligne : http://www.univ-rouen.fr/dyalang/glottopol, p. 2-3.

KERN, R. G., 1995, "Restructuring classroom interaction with networked computers: Effects on quantity and quality of language production”, Modern Language Journal 79(4), p. 457-476.

LAMY, M.-N., 2001, «L'étude d'une langue vivante assistée par ordinateur : réflexion collaborative sur l'objet d'apprentissage », in Bouchard, R. \& Mangenot, F. (eds.), p. 131-144.

MANGENOT, F., 2003, «Tâches et coopération dans deux dispositifs universitaires de formation à distance ", Apprentissage des langues et systèmes d'information et de communication Vol. $6, \mathrm{n}^{\circ} 1$, p. 109-125. Revue en ligne : http://alsic.org

MANGENOT, F., 2004, Analyse sémio-pragmatique des forums pédagogiques sur Internet, in Salaün, J.-M. \& Vandendorpe, C. (coord.), Les défis de la publication sur le Web: hyperlectures, cybertextes et méta-éditions, Villeurbanne, Presses de l'Enssib, p. 103-123.

MAngenOt, F., 2008a, «Pratiques pédagogiques instrumentées et propriétés des outils : le cas des forums », Sticef Vol. 15. Revue en ligne $:$ http://sticef.org

MANGENOT, F., 2008b, «La question du scénario de communication dans les interactions pédagogiques en ligne », in Sidir, M., Baron, G.-L., Bruillard, E. (eds.), 2008, Journées communication et apprentissage instrumenté en réseaux (Jocair 2008), Paris, Hermès, Lavoisier, p. 13-26.

MANGENOT, F., SALAM, P., 2010, «Quelles normes langagières dans les échanges pédagogiques en ligne ? Une étude de cas ", in Bertrand, O. et Schaffner, I. (eds.), "Quel français enseigner? La question de la norme dans l'enseignement/apprentissage », Palaiseau, Les Éditions de l'Ecole Polytechnique, p. 53-72.

MARCOCCIA, M., 2000, «Les smileys: une représentation iconique des émotions dans la communication médiatisée par ordinateur", in Les émotions dans les interactions communicatives, Presses universitaires de Lyon, p. 249-263.

Marcoccia, M., Gauducheau, N., 2007, «L'Analyse du rôle des smileys en production et en réception : un retour sur la question de l'oralité des écrits numériques », Glottopol 10, revue en ligne : http://www.univ-rouen.fr/dyalang/glottopol, p. 39-55. 
François Mangenot

Mourlhon-Dallies, F., 2007 «Communication électronique et genres du discours », Glottopol 10 , revue en ligne : http://www.univ-rouen.fr/dyalang/glottopol, p. 11-23.

O'Rourke, B., 2005, "Form-focused Interaction in Online Tandem Learning", CALICO Journal, Vol. $22, n^{\circ} 3$, p. $433-466$.

Pierozak, I., 2007, «Prendre internet pour terrain », Glottopol 10, Regards sur l'internet, dans ses dimensions langagières, Penser les continuités et discontinuités, revue en ligne: http://www.univ-rouen.fr/dyalang/glottopol, p. 4-10.

SOUBRIÉ, T., 2006, «Le blog : retour en force de la "fonction" auteur », in Sidir, M., Bruillard, É., Baron, G.-L. (eds.) Actes du colloque JOCAIR, 6-7 juillet 2006, Université de Picardie Jules Verne. pp. 292-309. http://edutice.archives-ouvertes.fr/edutice-00138462

SOUBrié, T., 2008, "Images de soi dans un blog professionnel d'enseignants stagiaires ", Apprentissage des langues et systèmes d'information et de communication Vol. 11. Revue en ligne: http://alsic.org

Souchier, E., Jeanneret, Y., Le Marec, J., 2003, Lire, écrire, récrire, Paris, Bibliothèque publique d'information.

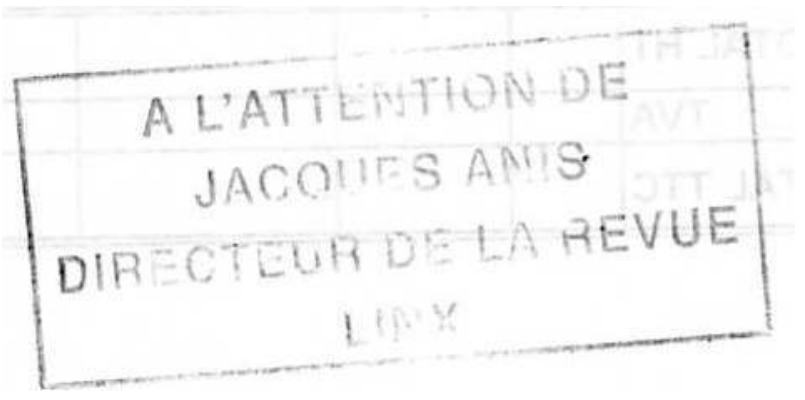

